\title{
ENERGIA DE REPOUSO DA CARGA ELÉTRICA E SPIN
}

\section{INTRODUÇÃO:}

A necèssidade de energia elétrica limpa e sustentável é uma demanda crescente da sociedade contemporânéa. Por isso, para finalizar este livro, apresentaremos outra aplicação tecnológica obtida a partir da Teoria Quântica da Gravidade. Dessa vez, contudo, a energia equivale a $\mathrm{E} \doteq \mathrm{m}_{0} \mathrm{c}^{2}$ e decorre do cessamento das propriedades intrínsecas e involuntárias do elétron. Diferentemente dos capítulos anteriores, este capítulo terá uma abordagem matemática maïs detalhada; porém, nada muito difícil. O motivo dessa diferença é que, neste capítulo, pretendemoś mostrar ao públịco leigo ou aos futuros cientistas como um físico teórico trabalha: manipulando equações -e leis físicas fundamentais da natureza. 


\section{METODOLOGIA:}

O método utilizado consiste em analisar um átomo que contém um único elétron. Além disso, sobre esse elétron, colocamos o nosso referencial inercial. Portanto, quem executa o movimento orbital é o próton. Esse fato (movimento do próton) justifica a ação de um campo magnético (B) sobre o elétron. A partir da interação do campo magnético (B) com o momento de dipolo magnético do spin do elétron $\left(\mu_{s}\right)$, deduzimos a equação da energia inercial associada à carga elétrica e ao spin. Tal energia é equivalente a $\mathrm{E}=\mathrm{m}_{0} \mathrm{c}^{2}$. O elétron em repouso é similar a um elétron livre em equilíbrio eletrostático. Por isso, a constante giromagnética é igual a $\gamma=1,76085977 \times 10^{11} \mathrm{C} . \mathrm{Kg}^{-1}$, e as outras constantes fundamentais são: carga elétrica $q=1,602176634 \times 10^{-19} \mathrm{C}$, fator g do spin: $g_{s}=2,00231930$ e velocidade da luz (c): $299792458 \mathrm{~m} \cdot \mathrm{s}^{-1}$.

\section{RESULTADOS E DISCUSSÕES:}

Em um átomo com um único elétron, a energia potencial orientacional $(\Delta \mathrm{E})$ do momento de dipolo magnético do spin do elétron $\left(\mu_{s}\right)$ interagindo com o campo magnético interno (B) é:

$$
\Delta E=-\mu_{s} \cdot \mathrm{B}(1)
$$


$\mu_{S}$ está em função do fator $g$ do $\operatorname{spin}\left(g_{S}\right)$, do momento angular intrínseco de spin (S), do magnéton de Bohr $\left(\mu_{b}\right)$ e da constante de Planck reduzida $(\hbar)$; isto é:

$$
\mu_{S}=-\frac{g_{s} \mu_{b}}{\hbar} S
$$

A componente de $\mu_{s}$ no eixo z pode assumir dois valores, pois $m_{s}=-1 / 2,+1 / 2$ :

$$
\mu_{S, z}=-g_{s} \mu_{b} m_{s}
$$

Em que $\mu_{b}$ é:

$$
\mu_{b}=\frac{q \hbar}{2 m}=0,927 \times 10^{-23} \mathrm{Am}^{2}
$$

A energia potencial orientacional $(\Delta \mathrm{E})$ é conservada, pois o ângulo entre os vetores $\mu_{S}$ e $\mathrm{B}$ é constante. $\mathrm{O}$ movimento de $\mu_{S}$ em torno de B é de precessão, também conhecido como precessão de Larmor. A frequência de Larmor $(\omega)$ está em função de B e da constante giromagnética $(\gamma)$ :

$$
\omega=2 \pi f=\gamma B
$$

O spin do elétron já era postulado na mecânica quântica de Schrödinger, mas foi o físico Paul Dirac, através da teoria quântica relativística, que mostrou que o elétron deveria ter spin e $\mu_{s}$.

Da Teoria da Relatividade Espacial, também obtemos a equivalência entre energia e massa de repouso $\left(m_{0}\right)$ : 


$$
E=m_{0} c^{2}(6)
$$

Uma pergunta fundamental é: "Existe equivalência entre energia (Equação 6) com a carga elétrica e o spin?" A resposta é sim, conforme mostraremos a seguir.

A Figura 1 ilustra o campo magnético (B) na direção z. Especificamente, a Figura 1-a mostra o ângulo $\theta$ formado entre o vetor $\mu_{s}$ e o eixo $z$, além da frequência angular de precessão de Larmor ( $\omega$ ) e da componente $\mu_{s, z}$ : O cone representa o produto escalar de $\mu_{s} . B$ no tempo, isto é, $\Delta \mathrm{E}$. Aqui, nós interpretamos que $\Delta \mathrm{E}$ equivale à energia cinética de precessão. Já na Figura 1-b, o vetor $\mu_{s}$ foi alinhado com a direção do campo magnético (B), ou seja, com a direção z. Por isso, o movimento de precessão cessa, e o cone de energia cinética de precessão $(\Delta \mathrm{E})$ é dissipado na geometria do espaço-tempo-energia na forma de um fóton.
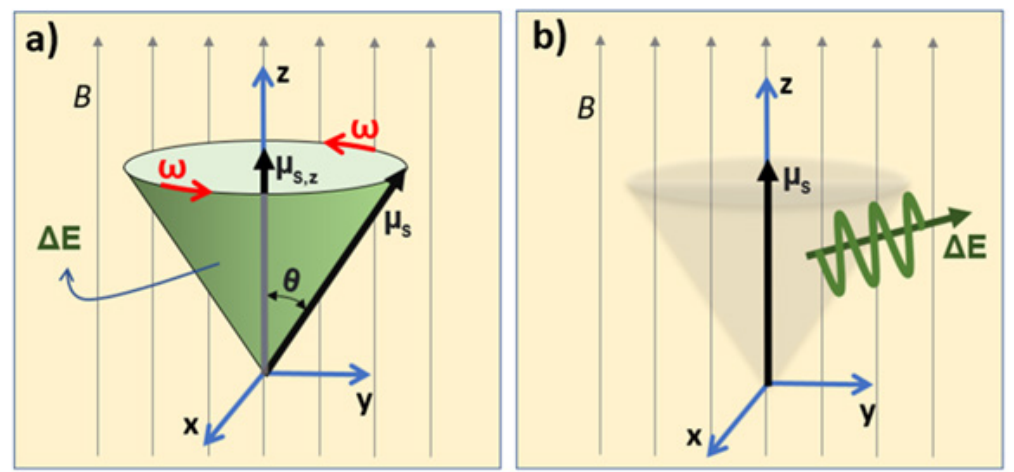

Figura 1 - a) interação do campo magnético (B) com vetor $\mu \mathrm{s}$, resultando no movimento de precessão de Larmor com frequência $\omega$. O cone representa a energia cinética associada ao movimento de precessão que é equivalente à energia potencial orientacional, isto é, $\Delta E$; b) o alinhamento do vetor us com a direção $z$ do campo magnético (B) promove o fim 
do movimento de precessão. A energia cinética associada à precessão do elétron é dissipada por um fóton.

A interação de $\mu_{s}$ com B foi descrita na Equação 1. Por isso, a componente $\mu_{s}$ na direção z torna-se:

$$
\mu_{s, z}=\mu_{s} \cos \theta \leftrightarrow \mu_{s}=\frac{\mu_{s, z}}{\cos \theta}
$$

Logo, $\Delta \mathrm{E}$ (Equação 1), na direção do eixo z, fica sendo:

$$
\Delta E_{z}=-\frac{\mu_{s, Z}}{\cos \theta} \cdot \mathrm{B}
$$

Devido ao fato de B e $\mu_{s, z}$ estarem na mesma direção, o ângulo será nulo $\left(\alpha=0^{\circ}\right)$. Portanto, o produto escalar de $\mu_{s, z} . \mathrm{B}\left(\mu_{s, z} B \cos \alpha\right.$, onde $\left.\cos 0^{\circ}=1\right)$ se resume em $\mu_{s, z} B$. Logo, a Equação 8 torna-se:

$$
\Delta E_{z}=-\frac{\mu_{s, z} B}{\cos \theta}
$$

Substituindo $\mu_{s, z}$ (Equação 3) na Equação 9, encontraremos:

$$
\Delta E_{z}=\frac{g_{s} \mu_{b} m_{s} B}{\cos \theta}
$$

Usando $\mu_{b}$ (Equação 4), $m_{s}=| \pm 1 / 2|, \quad \theta=60^{\circ}$ $\cos \theta=\frac{1}{2}$ ângulo de máxima otimização) na Equação 10, obtemos:

$$
\Delta E_{z}=\frac{g_{s} q \hbar B}{2 m}
$$


Da Equação 5, obtemos a expressão para o campo magnético B; por isso, substituindo B na Equação 11 e simplificando, temos:

$$
\Delta E_{z}=\frac{g_{s} q h}{2 m 2 \pi} \frac{2 \pi f}{\gamma}=\frac{g_{s}}{2 \gamma} q \frac{h f}{m}
$$

Agrupando $g_{s}$ e $2 \gamma$, obtemos a constante A, cuja unidade é $\mathrm{KgC}^{-1}$ :

$$
A=\frac{g_{s}}{2 \gamma}=5,68562964 \times 10^{-12}
$$

Substituindo, na Equação 12, a constante A e $h f / m$ por $c^{2}$ (já que $h f$ é energia, e a razão da energia pela massa de repouso é igual a $c^{2}$ ), encontramos a energia equivalente à massa, à carga elétrica e ao spin. Por isso, uol (Eureka!)

$$
\Delta E=A q c^{2}=m_{0} c^{2}=8.18710509 \times 10^{-14} \mathrm{~J}
$$

A energia inercial da carga elétrica e do spin do elétron é equivalente à energia cinética associada ao movimento de precessão de Larmor. Por isso, se $\mu_{s}$ (e não apenas $\mu_{s, z}$ ) alinhar com B, o movimento de precessão cessa. Então, a energia cinética de precessão é dissipada com intensidade $\mathrm{m}_{0} \mathrm{c}^{2}$ (Equação 14). Portanto, essa é a explicação quântica para a expressão deduzida por Albert Einstein $\left(E=m_{0} c^{2}\right.$, Equação 6)!

Outro resultado relevante é equivalência entre massa, carga elétrica e spin, conforme apresentado na Equação 15. Essa equação é válida quando há o alinhamento de $\mu_{s}$ e B. Porém, no estado fundamental (Equação 1 e 
Figura 1-a), a massa do elétron é formada parcialmente pela interação do campo de Higgs e pela interação entre carga elétrica e spin, pois $\theta \neq 0^{\circ}$ e $\mu_{s} \neq \mu_{s, z}$.

$$
m=\frac{g_{s}}{2 \gamma} q
$$

\section{CONCLUSÕES:}

A massa de repouso do elétron é parcialmente resultado do efeito quântico relativístico, isto é, $\gamma, g_{s}$ e q. $\mathrm{O}$ alinhamento de $\mu_{s}$ com o campo magnético B cessa o movimento de precessão, e a energia $\left(\mathrm{m}_{0} \mathrm{c}^{2}\right)$ é dissipada por meio de um fóton na geometria do espaço-tempo-energia. Esses resultados fomentam o desenvolvimento de pesquisas para a produção de energia limpa e sustentável, além de novos avanços científicos e tecnológicos obtidos naturalmente, por meio de Pesquisa e Desenvolvimento (P\&D). 